\title{
A commentary on a decrease in consumption of selective COX-2 inhibitors (coxibs) Comparison between Slovakia and Nordic countries
}

\author{
Hudec R, Kriska M, Tisonova J \\ Department of Pharmacology and Clinical Pharmacology, Faculty of Medicine, Comenius University, Bratislava, \\ Slovakia.dr.hudec@gmail.com
}

\begin{abstract}
Purpose: The aim of our work was to analyze utilization patterns in Slovakia and Nordic countries and to determine coxib risk perception among medical professionals.

Methods: The consumption of coxibs in Slovakia during 1999-2009 was analyzed. Data were obtained from the State Institute for Drug Control in SR. Obtained results were compared to data based on annual health statistics in Denmark, Finland and Norway. General Practitioners were asked on a perceived risk.

Results: We observed an increase of consumption in first years, followed with a marked decrease after year 2004. Slovak consumption was very small. Coxibs represented in Slovakia only a small part of totally prescribed non-steroidal anti-inflammatory drugs. They constituted only $1.44 \%$ in the year 2003 , whereas in Norway $41.2 \%$. $14 \%$ of respondents $(n=570)$ marked coxibs to be the safest analgesic drug.

Conclusion: Published data show that consumption of coxibs (rofecoxib was linked with serious thrombotic cardiovascular adverse effect) fall down markedly. Consumption data from 1999 until 2009 from Slovakia and three Nordic countries showed significant differences $(p<0.001)$. During the observed period, the utilization of coxibs in Slovakia was very small - a possible explanation is new drug, higher prize, doctor's habits (Tab. 2, Fig. 1, Ref. 11). Full Text in free PDF www.bmj.sk.

Key words: selective COX-2 inhibitors, coxibs, Slovakia, Nordic countries.
\end{abstract}

Search for an "aspirin-like drug", medicine, that would act anti-inflammatory without gastrotoxicity, lead to the synthesis of quantity of substances with different chemical structure. Around 30 of them reach the market over the past 30-40 years, but none was completely without gastrotoxicity (11).

Selective cyclooxygenase 2 (COX-2) inhibitors (coxibs) were approved for the relief of acute pain and symptoms of chronic inflammatory conditions such as osteoarthritis (OA) and rheumatoid arthritis (RA). Hypothesis was that COX-2 is the molecular target for the anti-inflammatory and analgesic effects of nonsteroidal anti-inflammatory drugs (NSAIDs). A corollary of it was that COX-2-selective inhibitors would retain the desirable effects of NSAIDs without some of their liabilities (e.g., gastrointestinal toxicity, which was described to COX-1 inhibition) (2).

They have similar pharmacological properties but a slightly improved gastrointestinal (GI) safety profile if compared to traditional nonsteroidal anti-inflammatory drugs (3).

Department of Pharmacology and Clinical Pharmacology, Faculty of Medicine, Comenius University, Bratislava, Slovakia

Address for correspondence: R. Hudec, MD, PhD, Dept of Pharmacology and Clinical Pharmacology, Faculty of Medicine, Comenius University, Sasinkova 4, SK-813 72 Bratislava, Slovakia.

Phone: +421.2.59357229, Fax: +421.2.59357508

Acknowledgement: We thank the State Institute for Drug Control in SR for providing data. Work was supported by grant VEGA No. 1/0135/09.
Lehmann and Beglinger in their article were more optimistic, declaring that gastrointestinal adverse drug reactions of NSAID have eminent economic fall and therefore coxibs, which had a comparative analgetic and antiinflammatory effect without adverse gastrointestinal effects designate an eminent addition to therapy (4).

Coxibs and possibly some NSAIDs also increase the risk of developing serious cardiovascular events, an effect which may depend on the drug, dose and duration of therapy (5).

The aim of our work was to analyze the utilization patterns in Slovakia and to compare them to Nordic countries. We also made a small questionnaire study aimed to determine coxib safety perception among GP's in Bratislava and rural cities.

\section{Methods}

The consumption of coxibs in Slovakia during 1999-2009 was analyzed. Data were obtained from the State Institute for Drug Control in SR. The analysis was based on the classical Defined Daily Doses (DDD) methodology according to the Anatomical Therapeutic Chemical Classification System (ATC). Expression of data as DDD per 1000 inhabitans per day enables a comparison of the active substance in different drug products.

Obtained results were compared to data based on annual health statistics in Finland, Norway and Denmark. We have chosen these countries as they represent countries with a high standard of pain 


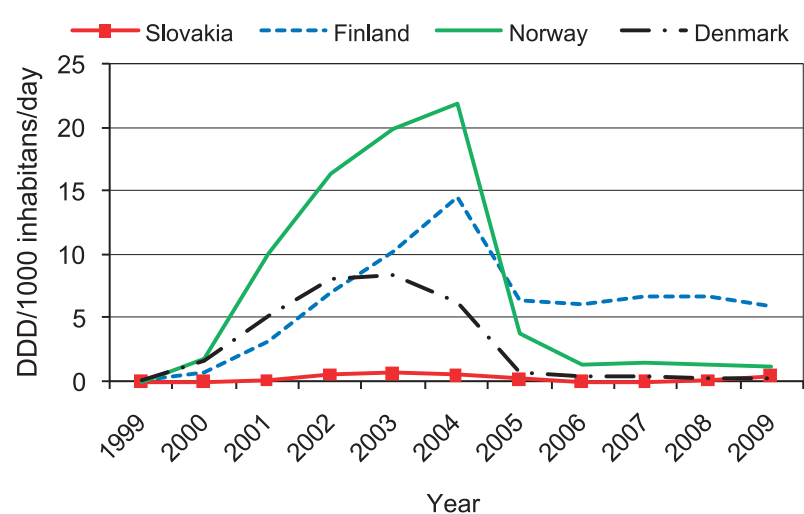

Fig. 1. Consumption trends of coxibs.

therapy and pharmacovigilance with critical appraisal of clinical efficacy and risk of drugs.

Participation of coxibs on the whole group consumption (ATC class M01A) in the years 1999-2009 was calculated. We have no patient oriented data so dose of coxib as well as duration of therapy could not be used.

We wanted to know perceived safety of coxibs. Slovak general practitioners (GPs) attending Continuing Medical Education (CME) lectures during 2004-2005, were invited to participate in our study. A mini interview was made with a simple question - "Which analgesic drug do you consider the safest?" This question had no predefined answers, so GP's wrote their own opinion.

In our mini interview, the respondents were divided into 2 groups. Group 1 consisted of doctors from Bratislava (capital city of Slovakia, $\mathrm{n}=245$ ) and group 2 consisted of GP's from 3 smaller rural cities (middle and eastern Slovakia with less than 200000 inhabitants, $n=325$ ). We tried to compare possible regional influences within Slovakia.

Results were statistically analyzed using the program Statgraphics Plus 5.1 with, Student's t-test used for consumption and chi squared test used for the interview. Differences were tested using an a level of 0.05 .

\section{Results}

Observed data are shown in Figure 1. There was a rapid increase of consumption in Nordic countries in first years, followed with a marked decrease after the year 2004. Slovak consumption was low, therefore changes in consumption were not so obvious.

Coxibs represented in Slovakia only a small part of totally prescribed non-steroidal anti-inflammatory drugs (Tab. 1). They constituted only $1.4 \%$ in the year 2003 , whereas in Norway $41.2 \%$.
Tab. 2. Results of mini interview with Slovak GPs - suggestions the safest analgesic drug. The mini interview was conducted before the publication on coxib's cardiovascular toxicity.

\begin{tabular}{lccc}
\hline $\begin{array}{l}\text { Generic name } \\
\text { of analgesic } \\
\text { drug }\end{array}$ & \multicolumn{2}{c}{ Percentual deal of answers (\%) } & $\mathrm{p}$ \\
\cline { 2 - 3 } & Group 1 (n=245) & Group 2 $(\mathrm{n}=325)$ & \\
\hline Coxibs & 19.21 & 10.15 & $<0.001$ \\
Tramadol & 16.75 & 19.93 & $-<0.05$ \\
Paracetamol & 12.81 & 19.55 & $<0.001$ \\
Diclofenac & 4.93 & 3.38 & $>0.05$ \\
Ibuprofen & 2.46 & 4.14 & $<0.05$ \\
Metamizol & 2.46 & 3.01 & $>0.05$ \\
Nimesulid & 0.99 & 5.64 & $<0.001$ \\
Aspirin & 0.49 & 1.50 & $>0.05$ \\
\hline
\end{tabular}

Respondents in the Group 1 marked coxibs to be the safest analgesic drug in $19.2 \%$, whereas in the Group 2 only $10.2 \%$ $(p<0.001)$ (Tab. 2). More than quarter of respondents wrote only NSAID without specification.

\section{Discussion}

New data support the critical opinion that even though selectively COX-2 inhibitors have minor gastro-intestinal complication like NSAID, they are not proper therapy for all patients.

The resolution to withdraw rofecoxib was conditional with preliminary results of the Adenomatous Polyp PRevention On Vioxx (APPROVE) trial comprising 2587 patients. Safety profile of rofecoxib was in centre of interest from publication of results of Vioxx GastroIntestinal Outcomes Research (VIGOR) trial - 8000 patients with rheumatoid arthritis, where group with rofecoxib had 4-fold higher incidence of cardiac infarction than the group with naproxen $(6,7)$.

European Medicines Agency (EMA) re-evalued coxib group - particularly celecoxib, etoricoxib, lumiracoxib, valdecoxib a parecoxib. Researchers found out an enhanced risk of cardiovascular adverse drug reactions and the majority of representatives were withdrawn from the market.

Currently two oral coxibs are available, celecoxib and etoricoxib, and one parenteral coxib, parecoxib. Lumiracoxib was withdrawn for hepatotoxicity, valdecoxib due to cardiovascular toxicity. Celecoxib has been on the market for the longest time and has the largest body of evidence. Moodley in his work wrote that in patients at risk for cardiovascular complications, both NSAIDs and coxibs have been shown to increase the risk of myocardial infarctions (MI), hypertension and heart failure (8).

Tab. 1. Participation of coxibs on whole group consumption (in \% of the total consumption of ATC class M01A).

\begin{tabular}{|c|c|c|c|c|c|c|c|c|c|c|c|}
\hline Year/contry & 1999 & 2000 & 2001 & 2002 & 2003 & 2004 & 2005 & 2006 & 2007 & 2008 & 2009 \\
\hline Slovakia & - & - & 0.19 & 1.21 & 1.44 & 1.22 & 0.49 & 0.05 & 0.05 & 0.19 & 0.86 \\
\hline Finland & 0.03 & 1.16 & 4.88 & 10.57 & 14.51 & 21.23 & 9.23 & 10.34 & 10.22 & 11.02 & 7.01 \\
\hline Norway & - & 5.33 & 24.94 & 35.73 & 41.22 & 43.23 & 9.93 & 3.73 & 3.63 & 3.13 & 2.63 \\
\hline Denmark & 0.03 & 3.12 & 9.45 & 14.09 & 13.97 & 10.57 & 1.14 & 0.67 & 0.56 & 0.46 & 0.41 \\
\hline
\end{tabular}


Newly approved drugs, in comparison to older drugs, are more often prescribed to patients who have not responded satisfactorily to established drugs or as first-line therapy to patients with a high baseline risk for the adverse outcomes (i.e. channeling). However, these patients are less likely to benefit from the prescribed drug and/or are more prone to adverse drug reactions. Therefore, it is difficult to unravel whether the observed risks or increases in risk of new drugs are real, i.e. related to the pharmacology, or whether these are related to selective prescribing to patients who are more susceptible to adverse events because of some underlying risk factor(s) (9).

The consumption data from the year 1999 till the year 2009 from Slovakia and three Nordic countries showed significant differences $(p<0.001)$. Utiliyation of coxibs in Slovakia was very low with a possible explanation of prescribing limitations, unsuccessful drug information as most influencing factors. Situation in other Central and Eastern European countries was similar (10).

We have documented a difference in risk perception between general practitioners in Bratislava and rural cities. Our mini interview was performed before the publication on possible cardiotoxicity. Limitation of the study should be doctor educations, age, years of practice, socio-economic status. Segment of the population receiving these drugs, mean age, gender distribution are also important influencing factors.

Further studies are needed to inform healthcare agencies and to encourage participation in the post-marketing safety studies declaring benefits over risks of drugs. Patients and professionals want much information about risks of medicines (11).

\section{References}

1. Pairet M, Van Ryn J (Eds). COX-2 Inhibitors. Basel, Birkhäuser Verlag 2004. 248 p.

2. Marnett LJ. The COXIB Experience: A Look in the Rear-View Mirror. Annu Rev Pharmacol Toxicol 2009; 49: 265-90.
3. Shi S, Klotz U. Clinical use and pharmacological properties of selective COX-2 inhibitors. Eur J Clin Pharmacol 2008; 64 (3): 233-252.

4. Lehmann FS, Beglinger C. Impact of COX-2 inhibitors in common clinical practice : a gastroenterologist's perspective. Curr Top Med Chem 2005; 5 (5): 449-464.

5. Lanas A, Hunt R. Prevention of anti-inflammatory drug-induced gastrointestinal damage: benefits and risks of therapeutic strategies. Ann Med 2006; 38 (6): 415-428.

6. Jüni P, Nartey L, Reichenbach S et al. Risk of cardiovascular events and rofecoxib: cumulative meta-analysis. Lancet 2004; 364: 2021-2029.

7. Konstantinopoulos PA, Lehmann DF. The cardiovascular toxicity of selective and nonselective cyclooxygenase inhibitors: comparisons, contrasts and aspirin confounding. J Clin Pharmacol 2005; 45 (7): 742-750.

8. Moodley I. Review of the cardiovascular safety of COXIBs compared to NSAIDS. Cardiovasc J Afr 2008; 19 (2): 102-107.

9. Layton D, Souverein PC, Heerdink ER et al. Evaluation of risk profiles for gastrointestinal and cardiovascular adverse effects in nonselective NSAID and COX-2 inhibitor users: a cohort study using pharmacy dispensing data in The Netherlands. Drug Saf 2008; 31 (2): 143-158.

10. Inotai A, Hankó B, Mészáros A. Trends in the non-steroidal anti-inflammatory drug market in six Central-Eastern European countries based on retail information. Pharmacoepidemiol Drug Saf 2010; 19 (2): 183-190.

11. Moore RA, Derry S, McQuay HJ et al. What do we know about communicating risk? A brief review and suggestion for contextualising serious, but rare, risk, and the example of cox-2 selective and non-selective NSAIDs. Arthritis Res Ther 2008; 10 (1): R20.

Received August 18, 2009. Accepted September 20, 2011. 